\title{
AEROMAGNETIC MAP OF THE NORTH BROOKFIELD QUADRANGLE WORCESTER COUNTY, MASSACHUSETTS
}

\author{
GEOPHYSICAL INVESTIGATIONS \\ MAP GP-666
}

